\title{
COMPARATIVE STUDY ON COMBINED EFFECT OF SILICA FUME AND STEEL FIBER REINFORCED CONCRETE OVER CONVENTIONAL CONCRETE.
}

\author{
Asst Professor, Civil Engineering Department, S G Balekundri Institute of Technology, \\ Belagavi, Karknataka, India. \\ Professor, Civil Engineering Department, S D M College of Engineering and Technology, \\ Dharwad, Karnataka, India.
}

\section{ABSTRACT}

The present investigation carried out on concrete due to the effect of silica fume with and without steel fibers on Portland pozzolana cement. In this study we used concrete mixes with silica fume of $20 \%$, with addition of crimped steel fibers of diameter $0.5 \mathrm{~mm}$ with aspect ratio of 60 , at various percentages as $0 \%, 0.5 \%, 1.0 \%$ and $1.5 \%, 2.0 \%$ by the volume of concrete on M 30 grade of concrete. The effect of mineral admixture (silica fume ) as cement replacement material with and without steel fibers on mechanical properties were analyzed and compared with normal concrete. In comparison, with normal concrete the replacement of $20 \%$ cement by silica fume showed $6 \%, 8.5 \%$ and 10.75 increase in compressive strength at 28 days of curing. The proposed model was found to have good accuracy in estimating at 28 days compressive strength, split tensile strength and flexural strength of concrete.

\section{INTRODUCTION:}

Concrete is a well known material used for construction. Concrete is a material which consists of cement, aggregates, water etc., Concrete is one of the most important materials and is unique among the major construction materials. FRC is a mix which consists of short and discrete fibers that is uniformly spread and randomly oriented in mix . when compared with the efficiency of FRC with conventional concrete, FRC has far superior efficiency because of its advantages and applications and its important area of requisition includes earthquake resistant structures, blast resistant structures, highway pavements, airports, mining and tunnel linings work,hydraulic structures and bridge deck overlays.

Addition of silica fume to concrete improves the durability of concrete and also in protecting the embedded steel from corrosion.

\section{OBJECTIVES OF THE STUDY}

The main objective of this experimental investigation as follows:

1. To achieve M30 grade of concrete for the mix.

2. To determine and study the characteristic properties of Steel fiber reinforced concrete with a Steel fiber content of $0.5 \%, 1.0 \%, 1.5 \%, 2.5 \%$ by volume of concrete.

3. To find the effect of silica fume as replacement of cement with fixed amount of replacement of 205 respectively different 5 of fibers on the strength of concrete.

4. The workability properties are studied for different blend concrete (i.e., plain concrete, silica fume blended concrete) with SF, with slump test.

5. The strength properties such as compressive strength, split tensile strength and flexural strength after 28 days of curing are studied and compared. 


\section{METHODOLOGY}

The following procedure is adopted for mixing of the specimens

- Calculated quantity of cement, sand and aggregate is weighed according to the mix proportion of 1:2.08:3.54 which correspond to M30 grade of concrete for w/c=0.45.

- First the cement and sand are dry mixed and then coarse aggregate is added and dry mixed.

- And then measured quantity of fiber of required $\%$ is added and mixed till all the fibers are dispersed firmly and uniformly.

- Then to this dry mix with fiber, add required quantity of water and the entire mix is mixed homogeneously.

- Similarly the same mixing procedure is adopted for other mix sets with silica fume by replacing cement by required quantity.

- Then this fresh concrete is placed into the moulds which is been then compacted by hand and by using table vibrator for compaction.

\section{EXPERIMENTAL RESULTS}

Slump test results

The slump test results for mono FRC with replacement of cement by silica fume by $20 \%$ respectively are given below:

\begin{tabular}{|c|c|c|c|c|}
\hline Sl No & Fiber Content & $\mathrm{H} 1$ (mm) & $\mathrm{H} 2(\mathrm{~mm})$ & $\begin{array}{l}\text { Slump } \\
\mathrm{H}=\mathrm{H} 1-\mathrm{H} 2(\mathrm{~mm})\end{array}$ \\
\hline \multicolumn{5}{|c|}{ PLAIN CONCRETE } \\
\hline 1 & $0 \%$ & 300 & 232 & 90 \\
\hline 2 & $0.5 \%$ SF & 300 & 246 & 85 \\
\hline 3 & $1.05 \mathrm{SF}$ & 300 & 252 & 80 \\
\hline 4 & $1.5 \% \mathrm{SF}$ & 300 & 260 & 75 \\
\hline \multicolumn{5}{|c|}{ REPLACEMENT OF CEMENT BY 20\% SILICA FUME } \\
\hline 5 & $0 \%$ & 300 & 230 & 93 \\
\hline 6 & $0.5 \% \mathrm{SF}$ & 300 & 238 & 89 \\
\hline 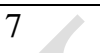 & $1.0 \% \mathrm{SF}$ & 300 & 246 & 80 \\
\hline 8 & $1.55 \mathrm{SF}$ & 300 & 240 & 78 \\
\hline
\end{tabular}

\section{STRENGTH TEST RESULTS}

COMPRESSIVE STRENGTH RESULT

\begin{tabular}{|c|c|c|c|c|}
\hline Sl No & $\begin{array}{l}\text { Fiber Content } \\
\%\end{array}$ & Load kN & $\begin{array}{l}\text { Compressive Strength } \\
=\text { P/A N } / \mathrm{mm}^{2}\end{array}$ & Average Result $\mathrm{N} / \mathrm{mm}^{2}$ \\
\hline \multicolumn{5}{|c|}{ PLAIN CONCRETE } \\
\hline \multirow[t]{3}{*}{1} & \multirow[t]{3}{*}{$0 \%$} & 680 & 30.22 & \multirow[t]{3}{*}{31.11} \\
\hline & & 720 & 32.00 & \\
\hline & & 700 & 31.11 & \\
\hline \multirow[t]{3}{*}{2} & \multirow[t]{3}{*}{$0.5 \%$ SF } & 735 & 32.60 & \multirow[t]{3}{*}{32.34} \\
\hline & & 720 & 32.00 & \\
\hline & & 730 & 32.44 & \\
\hline \multirow[t]{3}{*}{3} & \multirow[t]{3}{*}{$1.05 \mathrm{SF}$} & 750 & 33.33 & \multirow[t]{3}{*}{33.95} \\
\hline & & 760 & 33.77 & \\
\hline & & 780 & 34.66 & \\
\hline \multirow[t]{2}{*}{4} & \multirow[t]{2}{*}{$1.5 \% \mathrm{SF}$} & 850 & 37.77 & \multirow[t]{2}{*}{37.47} \\
\hline & & 820 & 36.44 & \\
\hline
\end{tabular}


DOI : https://dx.doi.org/10.26808/rs.ed.i8v4.05

International Journal of Emerging Trends in Engineering and Development

Issue 8, Vol.4 (June-July 2018)

Available online on http://www.rspublication.com/ijeted/ijeted_index.htm

ISSN 2249-6149

\begin{tabular}{|c|c|c|c|c|}
\hline & & 860 & 38.22 & \\
\hline \multirow[t]{3}{*}{5} & \multirow[t]{3}{*}{$2.0 \% \mathrm{SF}$} & 800 & 35.55 & \multirow[t]{3}{*}{35.18} \\
\hline & & 780 & 34.66 & \\
\hline & & 795 & 35.33 & \\
\hline \multicolumn{5}{|c|}{ REPLACEMENT OF CEMENT BY 20\% SILICA FUME } \\
\hline \multirow[t]{3}{*}{1} & \multirow[t]{3}{*}{$0 \%$} & 824 & 36.22 & \multirow[t]{3}{*}{36.35} \\
\hline & & 800 & 35.55 & \\
\hline & & 830 & 36.88 & \\
\hline \multirow[t]{3}{*}{2} & \multirow[t]{3}{*}{$0.5 \% \mathrm{SF}$} & 850 & 37.77 & \multirow[t]{3}{*}{38.21} \\
\hline & & 860 & 38.22 & \\
\hline & & 870 & 38.66 & \\
\hline \multirow[t]{3}{*}{3} & \multirow[t]{3}{*}{$1.0 \% \mathrm{SF}$} & 879 & 39.06 & \multirow[t]{3}{*}{38.92} \\
\hline & & 860 & 38.22 & \\
\hline & & 889 & 39.51 & \\
\hline \multirow[t]{3}{*}{4} & \multirow[t]{3}{*}{$1.5 \% \mathrm{SF}$} & 894 & 39.73 & \multirow[t]{3}{*}{40.05} \\
\hline & & 900 & 40.00 & \\
\hline & & 910 & 40.44 & \\
\hline \multirow[t]{3}{*}{5} & \multirow[t]{3}{*}{$2.0 \% \mathrm{SF}$} & 850 & 37.77 & \multirow[t]{3}{*}{37.99} \\
\hline & & 860 & 38.22 & \\
\hline & & 855 & 38.00 & \\
\hline
\end{tabular}

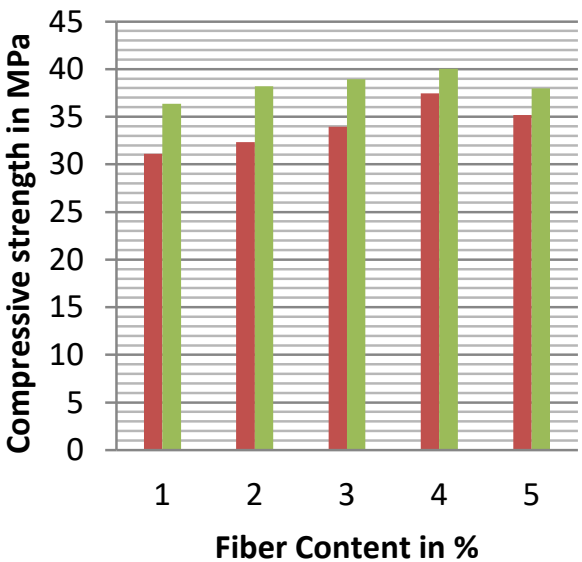

- Plain

Concrete

Silica Fume

Concrete

\section{SPLIT TENSILE STRENGTH}

\begin{tabular}{|c|c|c|c|c|}
\hline SI No & $\begin{array}{l}\text { Fiber Content } \\
\%\end{array}$ & Load kN & 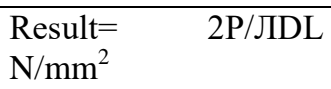 & $\begin{array}{l}\text { Average } \quad \text { Result } \\
\mathrm{N} / \mathrm{mm}^{2}\end{array}$ \\
\hline \multicolumn{5}{|c|}{ PLAIN CONCRETE } \\
\hline \multirow[t]{3}{*}{1} & \multirow{3}{*}{$0 \%$} & 160 & 2.26 & \multirow[t]{3}{*}{2.26} \\
\hline & & 150 & 2.12 & \\
\hline & & 170 & 2.40 & \\
\hline \multirow[t]{3}{*}{2} & \multirow[t]{3}{*}{$0.5 \% \mathrm{SF}$} & 210 & 2.97 & \multirow[t]{3}{*}{3.18} \\
\hline & & 240 & 3.39 & \\
\hline & & 230 & 3.18 & \\
\hline \multirow[t]{3}{*}{3} & \multirow[t]{3}{*}{$1.05 \mathrm{SF}$} & 260 & 2.97 & \multirow[t]{3}{*}{3.29} \\
\hline & & 270 & 3.18 & \\
\hline & & 265 & 3.74 & \\
\hline \multirow[t]{3}{*}{4} & \multirow[t]{3}{*}{$1.5 \% \mathrm{SF}$} & 285 & 4.03 & \multirow[t]{3}{*}{3.96} \\
\hline & & 275 & 3.89 & \\
\hline & & 280 & 3.96 & \\
\hline \multirow[t]{2}{*}{5} & \multirow[t]{2}{*}{$2.0 \% \mathrm{SF}$} & 260 & 3.67 & \multirow[t]{2}{*}{3.67} \\
\hline & & 255 & 3.60 & \\
\hline
\end{tabular}


DOI : https://dx.doi.org/10.26808/rs.ed.i8v4.05

International Journal of Emerging Trends in Engineering and Development Issue 8, Vol.4 (June-July 2018) Available online on http://www.rspublication.com/ijeted/ijeted_index.htm ISSN 2249-6149

\begin{tabular}{|c|c|c|c|c|}
\hline & & 265 & 3.74 & \\
\hline \multicolumn{5}{|c|}{ REPLACEMENT OF CEMENT BY 20\% SILICA FUME } \\
\hline \multirow[t]{3}{*}{1} & \multirow[t]{3}{*}{$0 \%$} & 182 & 2.57 & \multirow[t]{3}{*}{2.55} \\
\hline & & 175 & 2.47 & \\
\hline & & 185 & 2.61 & \\
\hline \multirow[t]{3}{*}{2} & \multirow[t]{3}{*}{$0.5 \% \mathrm{SF}$} & 240 & 3.39 & \multirow[t]{3}{*}{3.46} \\
\hline & & 230 & 3.25 & \\
\hline & & 265 & 3.74 & \\
\hline \multirow[t]{3}{*}{3} & \multirow[t]{3}{*}{$1.0 \% \mathrm{SF}$} & 278 & 3.93 & \multirow[t]{3}{*}{3.84} \\
\hline & & 270 & 3.81 & \\
\hline & & 269 & 3.80 & \\
\hline \multirow[t]{3}{*}{4} & \multirow[t]{3}{*}{$1.5 \% \mathrm{SF}$} & 285 & 4.03 & \multirow[t]{3}{*}{4.03} \\
\hline & & 280 & 3.96 & \\
\hline & & 290 & 4.10 & \\
\hline \multirow[t]{3}{*}{5} & \multirow[t]{3}{*}{$2.0 \% \mathrm{SF}$} & 265 & 3.74 & \multirow[t]{3}{*}{3.80} \\
\hline & & 275 & 3.89 & \\
\hline & & 270 & 3.81 & \\
\hline
\end{tabular}

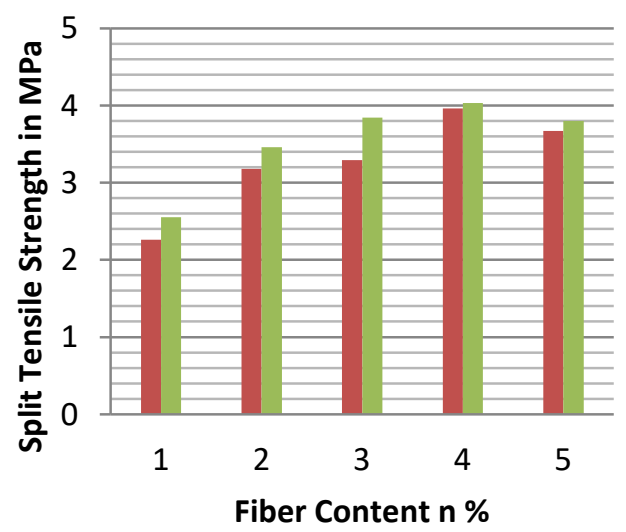

Plain

Concrete

Silica Fume

Concrete

FLEXURAL STRENGTH

\begin{tabular}{|c|c|c|c|c|}
\hline Sl No & Fiber Content $\%$ & Load kN & $\begin{array}{ll}\text { Result } & \mathrm{Pl} / \mathrm{BD}^{2} \\
\mathrm{~N} / \mathrm{mm}^{2} & \end{array}$ & Average Result N/mm 2 \\
\hline \multicolumn{5}{|c|}{ PLAIN CONCRETE } \\
\hline \multirow[t]{3}{*}{1} & \multirow[t]{3}{*}{$0 \%$} & 15 & 2.73 & \multirow[t]{3}{*}{2.97} \\
\hline & & 18 & 3.28 & \\
\hline & & 16 & 2.91 & \\
\hline \multirow[t]{3}{*}{2} & \multirow[t]{3}{*}{$0.5 \% \mathrm{SF}$} & 20 & 3.64 & \multirow[t]{3}{*}{3.72} \\
\hline & & 23 & 4.19 & \\
\hline & & 21 & 3.34 & \\
\hline \multirow[t]{3}{*}{3} & \multirow[t]{3}{*}{$1.05 \mathrm{SF}$} & 24 & 4.37 & \multirow[t]{3}{*}{4.61} \\
\hline & & 25 & 4.55 & \\
\hline & & 27 & 4.92 & \\
\hline \multirow[t]{3}{*}{4} & \multirow[t]{3}{*}{$1.5 \% \mathrm{SF}$} & 29 & 5.28 & \multirow[t]{3}{*}{5.50} \\
\hline & & 32 & 5.83 & \\
\hline & & 30 & 5.46 & \\
\hline \multirow[t]{3}{*}{5} & \multirow[t]{3}{*}{$2.0 \% \mathrm{SF}$} & 26 & 4.73 & \multirow[t]{3}{*}{4.73} \\
\hline & & 25 & 4.55 & \\
\hline & & 27 & 4.92 & \\
\hline
\end{tabular}




\begin{tabular}{|c|c|c|c|c|}
\hline \multicolumn{5}{|c|}{ REPLACEMENT OF CEMENT BY 20\% SILICA FUME } \\
\hline \multirow[t]{3}{*}{1} & \multirow[t]{3}{*}{$0 \%$} & 18 & 3.28 & \multirow[t]{3}{*}{3.14} \\
\hline & & 15 & 2.73 & \\
\hline & & 16 & 2.91 & \\
\hline \multirow[t]{3}{*}{2} & \multirow[t]{3}{*}{$0.5 \% \mathrm{SF}$} & 19 & 3.46 & \multirow[t]{3}{*}{3.45} \\
\hline & & 21 & 3.82 & \\
\hline & & 17 & 3.09 & \\
\hline \multirow[t]{3}{*}{3} & \multirow[t]{3}{*}{$1.0 \% \mathrm{SF}$} & 26 & 4.73 & \multirow[t]{3}{*}{4.91} \\
\hline & & 26 & 4.73 & \\
\hline & & 28 & 5.10 & \\
\hline \multirow[t]{3}{*}{4} & \multirow[t]{3}{*}{$1.5 \% \mathrm{SF}$} & 31 & 5.62 & \multirow[t]{3}{*}{5.63} \\
\hline & & 30 & 5.46 & \\
\hline & & 32 & 5.83 & \\
\hline \multirow[t]{3}{*}{5} & \multirow[t]{3}{*}{$2.0 \% \mathrm{SF}$} & 29 & 5.28 & \multirow[t]{3}{*}{5.22} \\
\hline & & 29 & 5.28 & \\
\hline & & 28 & 5.10 & \\
\hline
\end{tabular}

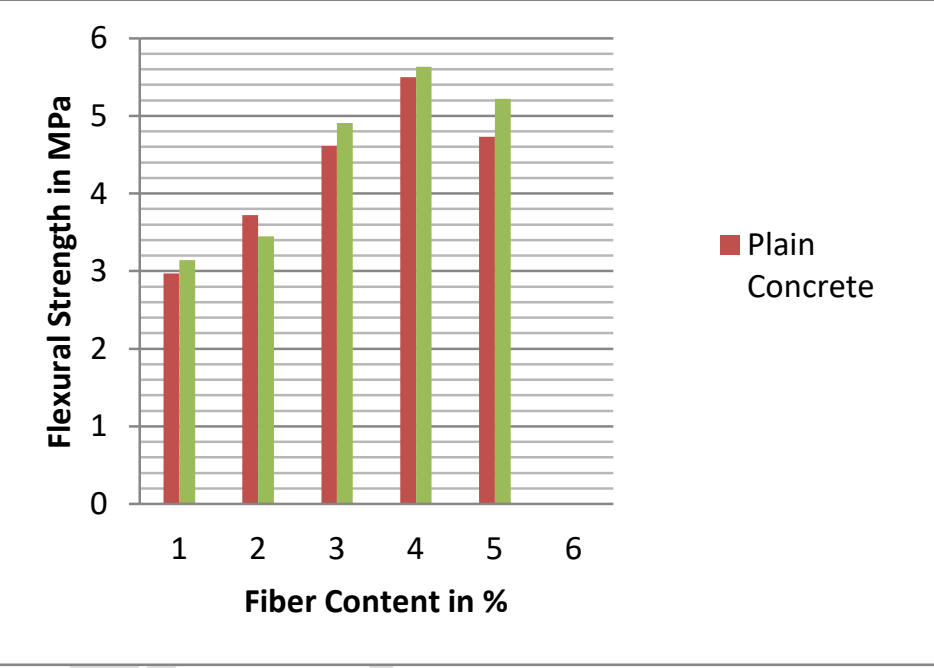

\section{CONCLUSION}

Following conclusions were drawn from the work carried out:

1. It is observed that the workability of steel fiber reinforced concrete gets reduced as the percentage of steel fibers increases.

2. Compressive strength goes on increasing by increase in steel fiber percentage upto the optimum value. The optimum value of fiber content of steel fiber content of steel fiber reinforced concrete is found to be $1.5 \%$.

3. The flexural strength of concrete goes on increasing with the increase in fiber content upto the optimum value. The optimum value for flexural strength of steel fiber reinforced concrete was found to be $1.5 \%$.

4. While testing the specimens, the plain cement concrete specimens have shown a typical crack propagation pattern which lead into splitting of beam in two piece geometry. But due to addition of fibers in concrete, cracks gets ceased which results into the ductile behavior of FSRC.

\section{REFERENCES}

I. Amit Rana. "Some studies on steel fiber reinforced concrete" International Journal of Emerging Technology and Advanced Engineering (IJETAE) Volume 3, Issue 1, January 2013, PP 120-217.

II. Indumati. B, V Gnanadevi . "An Experimental investigation on properties of steel fiber reinforced concrete with Silica fume" International Journal of Emerging Technology in 
Computer Science and Electronics (IJETCSE) Volume 13, Issue 4 - March 2015, PP 120123.

III. K Ramesh, Dr K Arunchalan , S Rooban Chakravarthy , "Experimental Investigation on Mechanical Properties of silica fume Concrete and silica fume fiber reinforced concrete" International journal of engineering research and applications (IJERA) Vol 3, Issue 3, May Jun 2013, PP 137-145.

IV. Mekala Prathap Reddy, Dr K Chandrashekhar Reddy “ Determination of mechanical properties of steel fiber reinforced concrete with mineral admixtures" International Journal and Magazine of Engineering Technology, Management and research (IJMETMR) Volume No: 2 (2015), Issue No 4: 4, April - 2015. PP 604-610. 